PROCEEDINGS OF THE

AMERICAN MATHEMATICAL SOCIETY

Volume 125, Number 11, November 1997, Pages 3371-3379

S 0002-9939(97)04012-4

\title{
ON THE CLASS OF NORM LIMITS OF NILPOTENTS
}

\author{
VASILE LAURIC \\ (Communicated by Palle E. T. Jorgensen)
}

\begin{abstract}
It is known that every operator on a Hilbert space $\mathcal{H}$ whose invariant subspace lattice is possibly $\{(0), \mathcal{H}\}$ is a norm-limit of a sequence of nilpotent operators. In this note we study properties of such approximating sequences.
\end{abstract}

\section{INTRODUCTION}

Let $\mathcal{H}$ denote a separable, infinite dimensional, complex Hilbert space and $\mathcal{L}(\mathcal{H})$ the algebra of all bounded linear operators on $\mathcal{H}$. If $T \in \mathcal{L}(\mathcal{H})$ we write, as usual, $\sigma(T)$ for the spectrum of $T$ and $\sigma_{e}(T)$ for the essential (i.e., Calkin) spectrum of $T$. Recall that an operator $T$ in $\mathcal{L}(\mathcal{H})$ is called quasitriangular [3] if there exists an increasing sequence $\left\{P_{n}\right\}_{n=1}^{\infty}$ of finite-rank projections converging strongly to $I_{\mathcal{H}}$ such that

$$
\left\|P_{n} T P_{n}-T P_{n}\right\| \rightarrow 0
$$

and $T$ is called biquasitriangular (notation: $T \in \mathcal{B Q T}(\mathcal{H})$ ) if both $T$ and $T^{*}$ are quasitriangular. In this note we study a certain subset $\mathcal{C}(\mathcal{H})$ of $\mathcal{L}(\mathcal{H})$, defined as follows.

Definition. An operator $T$ in $\mathcal{L}(\mathcal{H})$ belongs to the class $\mathcal{C}(\mathcal{H})$ if $T \in \mathcal{B Q T}(\mathcal{H})$, both $\sigma(T)$ and $\sigma_{e}(T)$ are connected subsets of the complex plane $\mathbf{C}$, and $0 \in \sigma_{e}(T)$.

A first reason that the class $\mathcal{C}(\mathcal{H})$ is interesting is that it has the following beautiful characterization, due to Apostol, Foiaş, and Voiculescu [2].

Theorem 1.1. An operator $T$ in $\mathcal{L}(\mathcal{H})$ belongs to $\mathcal{C}(\mathcal{H})$ if and only if there is a sequence $\left\{N_{k}\right\}_{k \geq 1}$ of nilpotent operators in $\mathcal{L}(\mathcal{H})$ such that $\left\|N_{k}-T\right\| \rightarrow 0$.

A second reason that the class $\mathcal{C}(\mathcal{H})$ is interesting is that the invariant subspace problem for operators in $\mathcal{L}(\mathcal{H})$ is equivalent to the invariant subspace problem for operators in $\mathcal{C}(\mathcal{H})$; cf. [4, Chapter 6$]$ :

Theorem 1.2. Every operator in $\mathcal{L}(\mathcal{H}) \backslash \mathcal{C}(\mathcal{H})$ has a nontrivial hyperinvariant subspace (n.h.s.).

For completeness, we briefly sketch the proof of this result. First, an operator $T$ in $\mathcal{L}(\mathcal{H})$ belongs to $\mathcal{B Q T}(\mathcal{H})$ if and only if for any complex number $\lambda$ such that $T-\lambda I_{\mathcal{H}}$ is a semi-Fredholm operator, the (Fredholm) index of $T-\lambda I_{\mathcal{H}}$ is

Received by the editors January 30, 1996 and, in revised form, June 26, 1996.

1991 Mathematics Subject Classification. Primary 47A15, 47A65.

Key words and phrases. Sequences of nilpotents, invariant subspaces.

(c)1997 American Mathematical Society 
equal to zero [1]. It follows trivially that every operator in $\mathcal{L}(\mathcal{H}) \backslash \mathcal{B} \mathcal{Q} \mathcal{T}(\mathcal{H})$ has a n.h.s. For, every such operator or its adjoint has point spectrum, and thus a n.h.s. Furthermore, if $\sigma(T) \neq \sigma_{e}(T)$, then $T$ is not a scalar and either $T$ or $T^{*}$ has point spectrum (and therefore a n.h.s.), so it suffices to consider those $T$ in $\mathcal{B Q T}(\mathcal{H})$ such that $\sigma(T)=\sigma_{e}(T)$. Moreover, if $\sigma(T)$ is not connected, then, by integrating around some connected component of $\sigma(T)$, one easily produces a nontrivial idempotent that commutes with the commutant of $T$, and thus a n.h.s. for $T$. Finally, since translation of an operator by a scalar preserves the existence of a n.h.s., one may suppose that $0 \in \sigma(T)$, which completes the sketch.

If we write $\mathcal{N}=\mathcal{N}(\mathcal{H})$ for the class of all nilpotent operators in $\mathcal{L}(\mathcal{H})$, and $\mathcal{S}^{-}$for the norm-closure of a subset $\mathcal{S}$ of $\mathcal{L}(\mathcal{H})$, then Theorem 1.2 can be paraphrased by saying that if $T \in \mathcal{L}(\mathcal{H})$ and $T$ is not known to have a n.h.s., then $T \in \mathcal{N}(\mathcal{H})^{-}=\mathcal{C}(\mathcal{H})$. Thus, for such a $T$ one knows that there exists a sequence $\left\{N_{k}\right\}_{k>1}$ of nilpotent operators from $\mathcal{L}(\mathcal{H})$ such that $\left\|N_{k}-T\right\| \rightarrow 0$.

The purpose of this note is to study the properties of such an approximating sequence $\left\{N_{k}\right\}_{k \geq 1}$ and, in particular, to derive as much structure as possible for this sequence, with the hope that nice properties of the sequence might, in the future, lead to invariant subspaces for the limit operator $T$.

\section{Approximating Sequences}

Let us fix an arbitrary operator $T$ in $\mathcal{N}(\mathcal{H})^{-}$and a sequence $\left\{N_{k}\right\}_{k \geq 1}$ of nilpotent operators in $\mathcal{L}(\mathcal{H})$ such that

$$
\left\|N_{k}-T\right\| \rightarrow 0 .
$$

Let $m_{k} \geq 1$ be the index of nilpotence of each $N_{k}$. For the purpose of showing that $T$ has a n.h.s., we may suppose that $\lim _{k}\left(m_{k}\right)=+\infty$. (For otherwise there exist a natural number $p$ and a subsequence $\left\{m_{k_{n}}\right\}_{n \geq 1}$ of $\left\{m_{k}\right\}$ such that $m_{k_{n}} \leq p$ for each $n \in N$. Consequently, since $\left\|T^{p}-N_{k}^{p}\right\| \rightarrow 0, T$ is nilpotent of index at most $p$ and has a n.h.s. for trivial reasons.) Moreover, there is obviously no loss of generality in supposing that the sequence $\left\{m_{k}\right\}$ is strictly increasing.

Lemma 2.1. Let $N$ be an operator in $\mathcal{N}(\mathcal{H})$ having index of nilpotence $m>1$. Then there exists a decomposition $\mathcal{H}=\mathcal{H}_{1} \oplus \cdots \oplus \mathcal{H}_{m}$ such that dim $\mathcal{H}_{i}=\aleph_{0}$, $i=1, \ldots, m$, and such that with respect to this decomposition the matrix of $N$ has the strictly lower triangular form

$$
N=\left(\begin{array}{cccc}
0 & 0 & \ldots & 0 \\
N_{2,1} & 0 & \ldots & 0 \\
\vdots & \vdots & \ddots & \vdots \\
N_{m, 1} & N_{m, 2} & \ldots & 0
\end{array}\right)
$$

(where, of course, for $1 \leq i, j \leq m, N_{i, j} \in \mathcal{L}\left(H_{j}, \mathcal{H}_{i}\right)$ ).

Proof. The proof is by induction on the index $m$ of nilpotence of $N$. We begin by noting that for an arbitrary $m>1$, we may write $\mathcal{H}=\mathcal{H}_{1}^{\prime} \oplus \cdots \oplus \mathcal{H}_{m}^{\prime}$, where $\mathcal{H}_{i}^{\prime}=\operatorname{Ker}\left\{\left(N^{*}\right)^{i}\right\} \ominus \operatorname{Ker}\left\{\left(N^{*}\right)^{i-1}\right\}=\left(\operatorname{Ran} N^{i-1}\right)^{-} \ominus\left(\operatorname{Ran} N^{i}\right)^{-}, i=1, \ldots, m$. 
Elementary considerations show that with respect to this decomposition of $\mathcal{H}$, the matrix of $N$ will have the form

$$
N=\left(\begin{array}{cccc}
0 & 0 & \ldots & 0 \\
N_{2,1}^{\prime} & 0 & \ldots & 0 \\
N_{3,1}^{\prime} & 0 & \ldots & 0 \\
\vdots & \vdots & \ddots & \vdots \\
N_{m, 1}^{\prime} & N_{m, 2}^{\prime} & \ldots & 0
\end{array}\right)
$$

where, of course, for $1 \leq i, j \leq m, N_{i, j}^{\prime}$ maps $\mathcal{H}_{j}^{\prime}$ into $\mathcal{H}_{i}^{\prime}$. (Easy examples show, however, that the dimension of some $\mathcal{H}_{i}^{\prime}$ may be finite.) For $m=2$, with respect to the decompositon $\mathcal{H}=\mathcal{H}_{1}^{\prime} \oplus \mathcal{H}_{2}^{\prime}$ the matrix of $N$ has the form

$$
N=\left(\begin{array}{cc}
0 & 0 \\
N_{2,1}^{\prime} & 0
\end{array}\right)
$$

where $\mathcal{H}_{1}^{\prime}=\mathcal{H} \ominus(\operatorname{Ran} N)^{-}=\operatorname{Ker} N^{*}$ (which is infinite dimensional, being the kernel of a nilpotent operator $)$, and $\mathcal{H}_{2}^{\prime}=(\operatorname{Ran} N)^{-} \subset \operatorname{Ker} N$. If $\operatorname{dim} \mathcal{H}_{2}^{\prime}=\aleph_{0}$, then setting $\mathcal{H}_{1}=\mathcal{H}_{1}^{\prime}$ and $\mathcal{H}_{2}=\mathcal{H}_{2}^{\prime}$ completes the proof in this case. On the other hand, if $N$ has finite rank, then we may write $\operatorname{Ker} N=\mathcal{K}_{1} \oplus \mathcal{K}_{2}$ where $\operatorname{dim} \mathcal{K}_{1}=\operatorname{dim} \mathcal{K}_{2}=\aleph_{0}$ and $\operatorname{Ran} N \subset \mathcal{K}_{2}$. Now set $\mathcal{H}_{1}=\mathcal{H} \ominus \mathcal{K}_{2}$ and $\mathcal{H}_{2}=\mathcal{K}_{2}$. Then $\mathcal{H}_{1} \subset \mathcal{H}_{1}^{\prime}$ and clearly $\mathcal{H}_{1}$ and $\mathcal{H}_{2}$ are infinite dimensional subspaces such that with respect to the decomposition $\mathcal{H}=\mathcal{H}_{1} \oplus \mathcal{H}_{2}, N$ has a matrix of the desired form.

Suppose now that the index of nilpotence $m$ of $N$ is greater than 2 and that the lemma has been proved for all nilpotent operators with index of nilpotence less than $m$. With respect to the decomposition $\mathcal{H}=\operatorname{Ker} N^{*} \oplus \operatorname{Ran} N^{-}$, the matrix of $N$ has the form

$$
N=\left(\begin{array}{cc}
0 & 0 \\
N^{\prime} & N^{\prime \prime}
\end{array}\right)
$$

It is easy to see that $N^{\prime \prime}$ is nilpotent of index $m-1$. Once again, one knows that $\operatorname{dim} \operatorname{Ker} N^{*}=\aleph_{0}$, and if $N$ is not of finite rank, we may apply the induction hypothesis to the operator $N^{\prime \prime}$, and conclude that there exists a decomposition Ran $N^{-}=\mathcal{H}_{2} \oplus \cdots \oplus \mathcal{H}_{m}$ such that each $\mathcal{H}_{i}, 2 \leq i \leq m$, is infinite dimensional and the matrix of $N^{\prime \prime}$ with respect to this decomposition has the form (1). By setting $\mathcal{H}_{1}=\operatorname{Ker} N^{*}$, we obtain the desired decomposition $\mathcal{H}=\mathcal{H}_{1} \oplus \cdots \oplus \mathcal{H}_{m}$. On the other hand, if $N$ has finite rank, then the same idea used in the case $m=2$ above, together with another application of the induction hypothesis, provides the desired decomposition.

Lemma 2.2. Let $T \in \mathcal{L}(\mathcal{H}, \mathcal{K})$ where $\mathcal{K}$ has dimension $\aleph_{0}$. Then for any $\varepsilon>0$ there are decompositions $\mathcal{H}=\mathcal{H}_{1} \oplus \mathcal{H}_{2}$ and $\mathcal{K}=\mathcal{K}_{1} \oplus \mathcal{K}_{2}$ and an operator $T^{\prime} \in$ $\mathcal{L}(\mathcal{H}, \mathcal{K})$ such that $\mathcal{H}_{1}, \mathcal{H}_{2}, \mathcal{K}_{1}, \mathcal{K}_{2}$ are infinite dimensional, $\left\|T-T^{\prime}\right\|<\varepsilon$, and the matrix of $T^{\prime}$ with respect to the above decompositions has the form

$$
T^{\prime}=\left(\begin{array}{cc}
* & T_{1,2}^{\prime} \\
* & *
\end{array}\right),
$$

where $T_{1,2}^{\prime}$ is an invertible operator in $\mathcal{L}\left(\mathcal{H}_{2}, \mathcal{K}_{1}\right)$. 
Proof. If $T$ is a compact operator, we choose arbitrary decompositions $\mathcal{H}=\mathcal{H}_{1} \oplus \mathcal{H}_{2}$ and $\mathcal{K}=\mathcal{K}_{1} \oplus \mathcal{K}_{2}$ where the $\mathcal{H}_{i}$ and $\mathcal{K}_{i}$ are infinite dimensional, $i=1,2$. With respect to these decompositions, the matrix of $T$ has the form

$$
T=\left(\begin{array}{cc}
* & T_{1,2} \\
* & *
\end{array}\right)
$$

where $T_{1,2}$ is compact. Therefore, for any $\varepsilon>0$ there exists $\lambda$ satisfying $0<\lambda<\varepsilon$ such that $T_{1,2}^{\prime}=T_{1,2}+\lambda$ is invertible, and hence one may take $T^{\prime}$ to have the same matrix as $T$ except that $T_{1,2}$ is replaced by $T_{1,2}^{\prime}$.

If $T$ is not compact, we consider the polar decomposition $T=U|T|$, and, of course, $|T|$ is not compact either. Let $E(\cdot)$ be the spectral measure of $|T|$. Then there exists $\theta>0$ such that the range of $E[\theta,+\infty)$ is an infinite dimensional subspace of $\mathcal{H}$, and the restriction of $T$ to the range $\mathcal{M}$ of $E[\theta,+\infty)$ is an invertible operator in $\mathcal{L}\left(\operatorname{Ran} E[\theta,+\infty)\right.$, Ran $\left.T_{\left.\right|_{\mathcal{M}}}\right)$. Now we decompose $\mathcal{M}=\mathcal{L}_{1} \oplus \mathcal{L}_{2}$ with both $\mathcal{L}_{1}$ and $\mathcal{L}_{2}$ infinite dimensional, and define $\mathcal{H}_{1}=\mathcal{L}_{2}^{\perp}, \mathcal{H}_{2}=\mathcal{L}_{2}, \mathcal{K}_{2}=T \mathcal{H}_{2}$, and $\mathcal{K}_{1}=\mathcal{K}_{2}^{\perp}$. It is clear that $T_{21}=T_{\left.\right|_{\mathcal{H}_{2}}}$ is invertible, and the lemma is proved.

Lemma 2.3. Let $N$ be an operator in $\mathcal{N}(\mathcal{H})$ with index of nilpotence $m>1$, and let $\varepsilon$ be any positive number. Then there exist $N^{\prime} \in \mathcal{N}(\mathcal{H})$ with index of nilpotence $p \geq 2 m$ and a decomposition $\mathcal{H}=\mathcal{K}_{1} \oplus \cdots \oplus \mathcal{K}_{p}$ such that $\left\|N-N^{\prime}\right\|<\varepsilon$, $\operatorname{dim} \mathcal{K}_{i}=\aleph_{0}, i=1, \ldots, p$, and the matrix of $N^{\prime}$ with respect to this decomposition has the strictly lower triangular form

$$
N^{\prime}=\left(\begin{array}{ccccc}
0 & 0 & \ldots & 0 & 0 \\
N_{2,1} & 0 & \ldots & 0 & 0 \\
\vdots & \ddots & \ddots & \vdots & \vdots \\
N_{p-1,1} & N_{p-1,2} & \ddots & 0 & 0 \\
N_{p, 1} & N_{p, 2} & \ldots & N_{p, p-1} & 0
\end{array}\right),
$$

where $N_{2,1}=0$ and $N_{i+1, i}$ is either invertible or zero for each $i=2, \ldots, p-1$.

Proof. We prove the lemma by induction on the index of nilpotence $m$ of $N$. First we assume that $m=2$. By Lemma 2.1, there exists a decomposition $\mathcal{H}=\mathcal{H}_{1} \oplus \mathcal{H}_{2}$ such that $\operatorname{dim} \mathcal{H}_{1}=\operatorname{dim} \mathcal{H}_{2}=\aleph_{0}$ and the matrix of $N$ with respect to this decomposition has the form

$$
N=\left(\begin{array}{cc}
0 & 0 \\
N_{2,1}^{\prime} & 0
\end{array}\right)
$$

Applying Lemma 2.2 to the operator $N_{2,1}^{\prime}: \mathcal{H}_{1} \rightarrow \mathcal{H}_{2}$, we get some infinite dimensional subspaces $\mathcal{K}_{1}, \ldots, \mathcal{K}_{4}$ such that $\mathcal{H}_{1}=\mathcal{K}_{1} \oplus \mathcal{K}_{2}, \mathcal{H}_{2}=\mathcal{K}_{3} \oplus \mathcal{K}_{4}$, and an operator $N_{2,1}^{\prime \prime}$ with $\left\|N_{2,1}^{\prime}-N_{2,1}^{\prime \prime}\right\|<\varepsilon$ such that with respect to these decompositions, the matrix of $N_{2,1}^{\prime \prime}$ has the form

$$
N_{2,1}^{\prime \prime}=\left(\begin{array}{cc}
* & N_{2,1} \\
* & *
\end{array}\right)
$$


where $N_{2,1}$ is invertible. With respect to the decomposition $\mathcal{H}=\mathcal{K}_{1} \oplus \mathcal{K}_{2} \oplus \mathcal{K}_{3} \oplus \mathcal{K}_{4}$ the matrix of $N$ has the form

$$
\left(\begin{array}{cccc}
0 & 0 & 0 & 0 \\
0 & 0 & 0 & 0 \\
* & N_{2,1} & 0 & 0 \\
* & * & 0 & 0
\end{array}\right)
$$

so the proof is complete in case $m=2$. Now suppose that $m>2$ and that the lemma has been proved for all nilpotent operators of index less than $m$. We apply Lemma 2.1 to $N$ to obtain a decomposition $\mathcal{H}_{1} \oplus \cdots \oplus \mathcal{H}_{m}$ and a matrix for $N$ with respect to this decomposition of the form (1). The operator $N_{\mid \mathcal{H}_{2} \oplus \cdots \oplus \mathcal{H}_{m}} \in \mathcal{L}\left(\mathcal{H}_{2} \oplus \cdots \oplus \mathcal{H}_{m}\right)$ is easily seen to be nilpotent of index $m-1$. Applying the induction hypothesis, we get a decomposition $\mathcal{H}_{2} \oplus \cdots \oplus \mathcal{H}_{m}=\mathcal{K}_{4}^{\prime} \oplus \mathcal{K}_{5} \oplus \cdots \oplus \mathcal{K} p$, where the direct sum on the right has $q$ summands (for some $q \geq 2 m-2$ ) all of which are infinite dimensional, such that the matrix of $N_{\left.\right|_{\mathcal{H}_{2} \oplus \cdots \oplus \mathcal{H}_{m}}}$ has the form

$$
\left(\begin{array}{cccccc}
0 & 0 & 0 & 0 & \ldots & 0 \\
0 & 0 & 0 & 0 & \ldots & 0 \\
* & N_{6,5} & 0 & 0 & \ldots & 0 \\
* & * & N_{7,6} & 0 & \ldots & 0 \\
\vdots & \vdots & \vdots & \vdots & \ddots & \vdots \\
* & * & * & * & \ldots & 0
\end{array}\right),
$$

where $N_{6,5}$ is invertible and each subdiagonal entry $N_{6+i, 5+i}$ is either 0 or is invertible. With respect to the decomposition $\mathcal{H}_{1} \oplus \mathcal{K}_{4}^{\prime} \oplus \mathcal{K}_{5} \oplus \cdots \oplus \mathcal{K}_{p}$, the matrix of $N$ has the form

$$
\left(\begin{array}{cccccc}
0 & 0 & 0 & 0 & \ldots & 0 \\
A_{2,1} & 0 & 0 & 0 & \ldots & 0 \\
* & 0 & 0 & 0 & \ldots & 0 \\
* & * & N_{6,5} & 0 & \ldots & 0 \\
\vdots & \vdots & \vdots & \vdots & \ddots & \vdots \\
* & * & * & * & \ldots & 0
\end{array}\right)
$$

where $A_{2,1}$ is the compresion of $N_{2,1}$ to the subspace $\mathcal{K}_{4}^{\prime}$. Next we apply a similar argument like that in the proof of the case $m=2$ to the operator

$$
N^{\prime \prime \prime}=\left(\begin{array}{cc}
0 & 0 \\
A_{2,1} & 0
\end{array}\right)
$$

and we get some infinite dimensional subspaces $\mathcal{K}_{1}, \mathcal{K}_{2}, \mathcal{K}_{3}, \mathcal{K}_{4}$ such that $\mathcal{H}_{1}=$ $\mathcal{K}_{1} \oplus \mathcal{K}_{2}, \mathcal{K}_{4}^{\prime}=\mathcal{K}_{3} \oplus \mathcal{K}_{4}$, and the matrix of $N^{\prime \prime \prime}$ with respect to the decomposition $\mathcal{H}_{1} \oplus \mathcal{K}_{4}^{\prime}=\mathcal{K}_{1} \oplus \cdots \oplus \mathcal{K}_{4}$ has the form (3). With respect to the decomposition $\mathcal{H}=\mathcal{K}_{1} \oplus \cdots \oplus \mathcal{K}_{p}$ with $p=q-1+4 \geq 2 m-3+4=2 m+1 \geq 2 m$, the matrix of $N$ has the desired form. Thus the lemma is proved.

Corollary 2.4. Let $N$ be an operator in $\mathcal{N}(\mathcal{H})$ with index of nilpotence $m>1$, and let $\varepsilon$ be any positive number. Then there exist $N^{\prime}$ in $\mathcal{N}(\mathcal{H})$ with index of nilpotence $p \geq 2 m$, and a decomposition $\mathcal{H}=\mathcal{K}_{1} \oplus \cdots \oplus \mathcal{K}_{p}$ such that $\left\|N-N^{\prime}\right\|<\varepsilon$, $\operatorname{dim} \mathcal{K}_{i}=\aleph_{0}, i=1, \ldots, p$, and the matrix of $N^{\prime}$ with respect to this decomposition 
has the strictly lower triangular form

$$
N^{\prime}=\left(\begin{array}{ccccc}
0 & 0 & \ldots & 0 & 0 \\
N_{2,1} & 0 & \ldots & 0 & 0 \\
\vdots & \vdots & \ddots & \vdots & \vdots \\
N_{p-1,1} & N_{p-1,2} & \ldots & 0 & 0 \\
N_{p, 1} & N_{p, 2} & \ldots & N_{p, p-1} & 0
\end{array}\right)
$$

where $N_{i+1, i}$, is invertible for $i=1, \ldots, p-1$.

Proof. Since in the previous lemma the subdiagonal operators $N_{i+1, i}$ are either invertible or zero, we can replace those operators $N_{i+1, i}$ which are zero by $(\varepsilon / 2) U_{i+1, i}$, where $U_{i+1, i}$ is a unitary operator from $\mathcal{H}_{i}$ onto $\mathcal{H}_{i+1}$ and thereby obtain the desired approximation.

Proposition 2.5. Let $N$ be a nilpotent operator such that the matrix representation of $N$ with respect to a decomposition $\mathcal{H}=\mathcal{H}_{1} \oplus \cdots \oplus \mathcal{H}_{m}, m>1$, has each subdiagonal entry $N_{i+1, i}, i=1, \ldots, m-1$, invertible. Then $N$ is unitarily equivalent to a nilpotent operator $N^{\prime}$ such that each subdiagonal entry $N_{i+1, i}^{\prime}, i=1, \ldots, m-1$, is a positive operator.

Proof. We prove the proposition by induction with respect to the index of nilpotence $m$. If $m=2$, then $N=\left(\begin{array}{cc}0 & 0 \\ N_{2,1} & 0\end{array}\right)$, with $N_{2,1}$ invertible. Then the polar decomposition $N_{2,1}=U_{2,1} P_{2,1}$ has $U_{2,1}$ unitary operator. Consider $U=\left(\begin{array}{cc}I & 0 \\ 0 & U_{2,1}\end{array}\right)$. Then $U^{*} N U=\left(\begin{array}{cc}0 & 0 \\ P_{2,1} & 0\end{array}\right)$. Suppose that the proposition is true for any index $k, 1<k \leq m$, and we prove that it remains true for $m+1$. Let $N=\left(\begin{array}{cc}0 & 0 \\ N_{1} & N_{2}\end{array}\right)$ be the matrix form of $N$ with respect to the decomposition $\mathcal{H}=\mathcal{H}_{1} \oplus \mathcal{H}_{1}^{\perp}$. The operator $N_{2}$ is nilpotent of index $m$, and thus by the induction hypothesis there exists a unitary operator $U^{\prime}$ on $\mathcal{H}_{2} \oplus \cdots \oplus \mathcal{H}_{m+1}$ such that

$$
U^{\prime *} N_{2} U^{\prime}=\left(\begin{array}{cccccc}
0 & 0 & 0 & \ldots & 0 & 0 \\
P_{3,2} & 0 & 0 & \ldots & 0 & 0 \\
* & P_{4,3} & 0 & \ldots & 0 & 0 \\
\vdots & \vdots & \ddots & \ddots & \vdots & \vdots \\
* & * & * & \ddots & 0 & 0 \\
* & * & * & \ldots & P_{m+1, m} & 0
\end{array}\right),
$$

with $P_{i+1, i}, i=2, \ldots, m$, positive operators. Consider $U^{\prime \prime}=I \oplus U_{2,1} \oplus I \oplus \cdots \oplus I$, where $N_{2,1}=U_{2,1} P_{2,1}$ is the polar decomposition of the invertible operator $N_{2,1}$. Then

$$
U^{\prime \prime *}\left(I \oplus U^{\prime}\right)^{*} N\left(I \oplus U^{\prime}\right) U^{\prime \prime}
$$

has the desired matrix form, so the proof is complete.

The following is our main result on the structure of approximating sequences of nilpotents. 
Theorem 2.6. Let $T$ be an operator in $\mathcal{N}(\mathcal{H})^{-} \backslash \mathcal{N}(\mathcal{H})$. Then there exist a sequence $\left\{N_{k}\right\}_{k \geq 1}$ of nilpotent operators, a strictly increasing sequence $\left\{m_{k}\right\}$ of positive integers, and a sequence $\left\{\mathcal{H}=\mathcal{K}_{1}^{(k)} \oplus \cdots \oplus \mathcal{K}_{m_{k}}^{(k)}\right\}$ of decompositions of $\mathcal{H}$ such that

(a) $m_{k}$ is the index of nilpotence of $N_{k}$,

(b) $\left\|N_{k}-T\right\| \rightarrow 0$,

(c) $\operatorname{dim} \mathcal{K}_{i}^{(k)}=\aleph_{0}$ for each positive integer $k$, and each $i=1, \ldots, m_{k}$,

(d) the elements $\left(N_{k}\right)_{i+1, i}, 1 \leq i \leq m_{k}-1$, of the matrix $N_{k}$ corresponding to the decomposition $\mathcal{H}=\mathcal{K}_{1}^{(k)} \oplus \cdots \oplus \mathcal{K}_{m_{k}}^{(k)}$ are invertible positive operators,

(e) $\bigcup_{k=1}^{\infty} \operatorname{Ran} N_{k} \neq \mathcal{H}$.

Proof. The first four conclusions of this corollary come directly from the previous lemmas. To prove (e), note that it follows easily from (d) that for each positive interger $k$, the range of $N_{k}$ is the subspace $(0) \oplus \mathcal{K}_{2}^{(k)} \oplus \cdots \oplus \mathcal{K}_{m_{k}}^{(k)}$ which is a proper subspace of $\mathcal{H}$ (necessarily nowhere dense in $\mathcal{H}$ ). By the Baire category theorem, $\bigcup_{k=1}^{\infty} \operatorname{Ran} N_{k} \neq \mathcal{H}$.

In the next proposition we show that the nilpotent operators $N_{k}$ appearing above are all similar to operators acting on a direct sum of finite number of copies of $\mathcal{H}$ and having matrices of the form

$$
J=\left(\begin{array}{ccccc}
0 & 0 & 0 & \ldots & 0 \\
I & 0 & 0 & \ldots & 0 \\
0 & I & 0 & \ldots & 0 \\
\vdots & \vdots & \vdots & \ddots & \vdots \\
0 & 0 & 0 & \ldots I & 0
\end{array}\right) .
$$

where each entry on the first subdiagonal of $J$ is the identity operator. Such a matrix is called a Jordan block [5]. More precisely, we have the following.

Proposition 2.7. Let $N \in \mathcal{L}\left(\mathcal{H}=\mathcal{H}_{1} \oplus \cdots \oplus \mathcal{H}_{m}\right)$ be a nilpotent operator of index $m>1$ of the form (4) where each $N_{i+1, i}$ is invertible. Then there exist an invertible operator $S$ from $\mathcal{H}^{(m)}$ (the direct sum of $m$ copies of $\mathcal{H}$ ) to $\mathcal{H}$ and a Jordan block operator $J$ in $\mathcal{L}\left(\mathcal{H}^{(m)}\right)$ as in (5), such that $S^{-1} N S=J$.

Proof. The proof is by induction on the index of nilpotence $m$. If $m=2$, then

$$
N=\left(\begin{array}{cc}
0 & 0 \\
N_{2,1} & 0
\end{array}\right)
$$

where $N_{2,1}$ is invertible in $\mathcal{L}\left(\mathcal{H}_{1}, \mathcal{H}_{2}\right)$. Let $\mathcal{H}_{1}$ and $\mathcal{H}_{2}$ be identified with $\mathcal{H}$ via Hilbert space isomorphisms. Then $N$ is unitarily equivalent to an operator $N^{\prime} \in$ $\mathcal{L}\left(\mathcal{H}^{(2)}\right)$ whose matrix has the form $\left(\begin{array}{cc}0 & 0 \\ M & 0\end{array}\right)$ with $M$ invertible. Then

$$
\left(\begin{array}{cc}
M & 0 \\
0 & I
\end{array}\right)\left(\begin{array}{cc}
0 & 0 \\
M & 0
\end{array}\right)\left(\begin{array}{cc}
M^{-1} & 0 \\
0 & I
\end{array}\right)=\left(\begin{array}{ll}
0 & 0 \\
I & 0
\end{array}\right)
$$

Next suppose that $m \geq 3$ and that the conclusion of Lemma 2.6 is true for any nilpotent operator with index less than $m$. Let $N^{\prime}$ be a nilpotent operator of index $m$ with matricial form (4) and satisfying the hypothesis of the lemma. As above, we can identify $\mathcal{H}_{1}, \mathcal{H}_{2}, \ldots, \mathcal{H}_{m}$ with $\mathcal{H}$. Then $N$ is unitarily equivalent to $N^{\prime} \in$ 
$\mathcal{L}\left(\mathcal{H}^{(m)}\right)$ whose matrix has the form

$$
N^{\prime}=\left(\begin{array}{ccccc}
0 & 0 & \ldots & 0 & 0 \\
N_{2,1}^{\prime} & 0 & \ldots & 0 & 0 \\
\vdots & \vdots & \ddots & \vdots & \vdots \\
N_{m, 1}^{\prime} & N_{m, 2}^{\prime} & \ldots & N_{m, m-1}^{\prime} & 0
\end{array}\right)
$$

with $N_{2,1}^{\prime}, N_{3,2}^{\prime}, \ldots, N_{m, m-1}^{\prime}$ invertible. By applying the induction hypothesis to the $(m-1) \times(m-1)$ matrix that is the upper left-hand corner of $N^{\prime}$, say $N^{\prime \prime}$, we get an invertible operator $S^{\prime}$ in $\mathcal{L}\left(\mathcal{H}^{(m-1)}\right)$ such that $S^{\prime-1} N^{\prime \prime} S^{\prime}=J^{\prime}$, where $J^{\prime}$ is the $(m-1) \times(m-1)$ Jordan block. Considering $S_{1}=\left(\begin{array}{cc}S^{\prime} & 0 \\ 0 & I\end{array}\right)$ an invertible operator in $\mathcal{L}\left(\mathcal{H}^{(m)}\right)$, then

$$
S_{1}^{-1} N^{\prime} S_{1}=\left(\begin{array}{ccccc}
0 & 0 & \ldots & 0 & 0 \\
I & 0 & \ldots & 0 & 0 \\
\vdots & \vdots & \ddots & \vdots & \vdots \\
0 & 0 & \ldots & 0 & 0 \\
M_{1} & M_{2} & \ldots & M_{m-1} & I
\end{array}\right)
$$

with $M_{m-1}$ invertible. (We should point here that $M_{m-1}$ is invertible because of the lower triangular matrix form of $S^{\prime}$.) We put now

$$
S_{2}=\left(\begin{array}{ccccc}
I & 0 & \ldots & 0 & 0 \\
0 & I & \ldots & 0 & 0 \\
\vdots & \vdots & \ddots & \vdots & \vdots \\
0 & 0 & \ldots & I & 0 \\
0 & M_{1} & \ldots & M_{m-2} & I
\end{array}\right)
$$

Then

$$
S_{2}^{-1}\left(S_{1}^{-1} N^{\prime} S_{1}\right) S_{2}=\left(\begin{array}{ccccc}
0 & 0 & \ldots & 0 & 0 \\
I & 0 & \ldots & 0 & 0 \\
\vdots & \vdots & \ddots & \vdots & \vdots \\
0 & 0 & \ldots & I & 0 \\
0 & 0 & \ldots & M_{m-1} & 0
\end{array}\right) .
$$

Finally, we take $S_{3}=I \oplus \cdots \oplus I \oplus M_{m-1} \in \mathcal{L}\left(\mathcal{H}^{(m)}\right)$. After a computation we get $S_{3}^{-1}\left[S_{2}^{-1}\left(S_{1}^{-1} N^{\prime} S_{1}\right) S_{2}\right] S_{3}=J$, where $J$ is the $m \times m$ Jordan block and $S_{1} S_{2} S_{3}$ has lower triangular matrix form. Thus the proof is done.

We write $\mathcal{H}=\mathcal{K}_{1}+\cdots+\mathcal{K}_{t}$ to mean that $\mathcal{H}$ is the (not necessarily orthogonal) direct sum of the subspaces $\mathcal{K}_{i}$.

Corollary 2.8. Let $T$ be an operator in $\mathcal{N}(\mathcal{H})^{-} \backslash \mathcal{N}(\mathcal{H})$. Then there exist a sequence $\left\{N_{k}\right\}_{k \geq 1}$ of nilpotent operators, a strictly increasing sequence $\left\{m_{k}\right\}$ of positive integers, and a sequence $\left\{\mathcal{H}=\mathcal{K}_{1}^{(k)} \dot{+} \cdots \dot{+} \mathcal{K}_{m_{k}}^{(k)}\right\}$ of direct sum decompositions of $\mathcal{H}$ with (a), (b), (c), (e) as in Theorem 2.6 and also $\left(\mathrm{d}^{\prime}\right)$ each matrix $N_{k}$ is a Jordan block.

This note raises some problems that would seem to be interesting:

1) Can one use the properties of an approximating sequence of nilpotents to establish the existence of an invariant subspace for the limit operator? 
$\left.1^{\prime}\right)$ Suppose $T$ is the limit of a sequence of Jordan block matrices. Does $T$ have a n.i.s?

2) Can additional properties of an approximating sequence of nilpotents be deduced when a) $T$ is quasinilpotent? b) $T=N+K \in \mathcal{N}(\mathcal{H})^{-}$, where $N$ is normal and $K$ is compact?

\section{REFERENCES}

1. C. Apostol, C. Foiaş, and D. Voiculescu, Some results on non-quasitriangular operators. IV, Rev. Roumaine Math. Pures Appl. 18 (1973), 159-181. MR 48:12109a

2. C. Apostol, C. Foiaş, and D. Voiculescu, On the norm-closure of nilpotents. II, Rev. Roumaine Math. Pures Appl. 19 (1974), 549-557. MR 54:5876

3. P. R. Halmos, Quasitriangular operators, Acta Sci. Math. (Szeged) 29 (1968), 283-293. MR 38:2627

4. C. Pearcy, Some recent developments in operator theory, CBMS Regional Conf. Ser. in Math. No 36, Amer. Math. Soc., Providence (1978). MR 58:7120

5. L. R. Williams, Similarity invariants for a class of nilpotent operators, Acta Sci. Math.(Szeged) 38 (1976), 423-428. MR 55:3832

Department of Mathematics, Texas A\&M University, College Station, Texas 77843

E-mail address: lauric@math.tamu.edu 